\title{
MAGNITUDES DENDROMÉTRICAS DE CUATRO POBLACIONES DE ALGARROBO BLANCO (PROSOPIS ALBA GRISEB.) DE DIFERENTES EDADES
}

\author{
Bender, A. ${ }^{1 ;}$ Araujo, J. ${ }^{1}$ Perreta, m. ${ }^{2} \&$ Moglia, J. ${ }^{3}$
}

\begin{abstract}
RESUMEN
Magnitudes dendrométricas fueron analizadas en cuatro poblaciones de Prosopis alba (algarrobo blanco) provenientes de poblaciones naturales y cultivadas. El objetivo fue evaluar las características dendrométricas en árboles de diferentes edades a fin de describir el comportamiento en distintos estadios de crecimiento y establecer relaciones alométricas en las mismas.

Las variables medidas fueron: diámetro, altura total, altura de fuste, ancho de copa, rectitud, sanidad de fuste y vitalidad de copa.

La correlación entre diámetro y altura mediante una ecuación potencial mostró un coeficiente satisfactorio $\left(\mathrm{R}^{2}=0.92\right)$ cuando se analizaron las cuatro poblaciones en conjunto. Las mejores características cualitativas se observaron en las poblaciones cultivadas.

La utilización de plantas jóvenes permitió tener valores del extremo inferior de las curvas de crecimiento, mientras que los datos del bosque nativo favorecieron el análisis de árboles de mayores dimensiones. Es necesario ampliar la cantidad de individuos de diferentes poblaciones para obtener ecuaciones más precisas de P. alba.

Palabras clave: Prosopis alba, magnitudes dendrométricas, relaciones alométricas, incremento anual.
\end{abstract}

\section{SUMMARY}

Dendrometric parameters of four populations of white mesquite (Prosopis alba Griseb.) of different ages.

Dendrometric parameters of four white mesquite populations (Prosopis alba) of different ages were analyzed. Variables measured were: diameter, total height, trunk height and crown width, trunk straightness, trunk health, and crown vitality. The correlation between diameter and total height through a potential equation showed a satisfac-

1.- Cátedra de Dasonomía - Facultad de Ciencias Agrarias, Universidad Nacional del Litoral. Kreder 2805. (3080) Esperanza, provincia de Santa Fe. Email: adriangbender@gmail.com

2.- Cátedra de Morfología Vegetal - (CONICET/Facultad de Ciencias Agrarias- UNL)

3.- Cátedra Dendrología- Facultad de Ciencias Forestales (UNSE).

Manuscrito recibido el 5 de febrero de 2015 y aceptado para su publicación el 8 de junio de 2015. 
A. Bender et al.

tory coefficient $(\mathrm{R} 2=0.92)$ when the four populations were analyzed together. The best qualitative features were observed in cultivated populations. The utilization of young plants allowed obtaining values at the lowest end of growth curves, meanwhile thenative forest data promoted the analysis of higher size trees. In orderto procure a more accurate equation for P. alba, it is necessary to extend the number of individuals in the sampling of every population.

Key words: White mesquite, dendrometric parameters, allometric relationships, annual increments.

\section{INTRODUCCIÓN}

Prosopis alba es uno de los árboles de área geográfica más amplia en Argentina (Tortorelli, 2009). Al igual que otras especies del género presenta gran importancia ecológica y ductilidad (Giménez et al., 2006); mostrando potencial para ser usada en la restauración de áreas degradadas (Taleisnik \& Lauestein, 2011). Actualmente es una de las maderas nativas de mayor uso (Galera, 2000; Tortorelli, 2009) y dado su potencial forestal y maderero constituye un importante recurso para los pueblos de zonas áridas y semiáridas (Giménez et al., 1998; 2006).

En la actualidad gran parte de la superficie originalmente ocupada por bosques de algarrobo se ha perdido a tasa creciente en el tiempo (Palacios y Brizuela, 2005). Esto es debido a dos causas principales: la deforestación y el uso para la industria. Las tasas de desforestación registradas en las últimas décadas en función fundamentalmente del avance de la agricultura, han eliminado gran parte del bosque nativo (Coirini, 2011). El desmonte recrudeció recientemente con la rápida expansión agrícola, proceso que favoreció la desaparición de una alta proporción de bosques de algarrobo (Arturi, 2005). Con respecto al uso industrial, la industria mueblera, ha realizado una extracción selectiva de algarrobos a límites cercanos a la extinción local de individuos maderables (Palacios y Brizuela, 2005), sobre todo en áreas cercanas a los núcleos muebleros. Si bien su madera es sumamente aprovechada es insuficiente la investigación científica desarrollada que permita establecer turnos adecuados de aprovechamiento (Giménez et al., 2001). En Argentina, en general, son escasos los antecedentes sobre estudios de crecimiento de especies leñosas nativas (Araujo et al., 2007).

La información referida al crecimiento es básica para la elaboración y aplicación de un plan de manejo forestal (Juárez de Galíndez, 2005), para determinar adecuadamente los ciclos de corta y para regular la producción del bosque (Araujo et al., 2007; Bormann \& Berlyn, 1983).

Una explotación forestal racional implica una serie de conocimientos precisos acerca de la biología, comportamiento según la edad y ritmo de crecimiento de las especies (Bormann \& Berlyn, 1983). El principal problema para iniciar un proyecto de reforestación, o forestación en regiones aptas para el cultivo de algarrobos, es la poca información relativa al crecimiento en diámetro, altura y las variables que de ellas se derivan para lograr un manejo adecuado (Palacios \& Brizuela, 2005; Juárez de Galíndez, 2005). Husch et al. (1982) señalan que la altura total y el diámetro son 